\title{
Percutaneous Transforaminal Endoscopic Discectomy in The Treat- ment of Lumbar Disc Herniation: Effects on Early Rehabilitation
}

\author{
Abudourexiti·Tuerhognjiang*, Rehaman·Sulaiman, Xiang-yu Meng, Liang Ma, Yun-tao Liu, \\ Chuan-yu He, Bing Liang \\ Department of Minimally Invasive Spine Therapy, The Sixth Affiliated Hospital of Xinjiang Medical University. Urumchi, 830001, China.
}

\begin{abstract}
Objective: The aim of this research was to evaluate the early rehabilitation and complications of percutaneous transforaminal endoscopic discectomies (PTEDs) in the treatment of lumbar disc herniations.

Methods: From July 2015 to January 2017, ninety-one patients with lumbar disc herniations who underwent PTEDs were retrospectively enrolled. During the six month follow-up period, a visual analogue scale (VAS) and the Oswestry Disability Index (ODI) were used to evaluate the preoperative and postoperative ( 3 days, 3 months and 6 months) disturbance indexes. The Macnab criteria were used to evaluate the clinical efficacy. The operation time, intraoperative blood loss, average length of the hospital stay, postoperative recurrence rate, and complications were observed and recorded.

Result: The VAS and ODI scores for the lumbocrural pain after the surgery were significantly lower than those before the surgery $(p<0.05)$. There were no significant differences in the lower back pain VAS scores on the postoperative 3rd day, 3rd month, and 6th month ( $p>0.05)$. The leg pain VAS score on the postoperative 3rd day was significantly lower than that during the postoperative 3rd month $(\mathrm{p}<0.05)$. The lower back pain VAS score during the postoperative 6th month was lower than that on the postoperative 3rd day, with no statistical significance $(p>0.05)$.

Conclusion: Based on the results of this study, PTEDs for the treatment of lumbar disc herniations are safe and effective, with less trauma and complications. In addition, the postoperative rehabilitation time was markedly shortened.

Keywords: percutaneous transforaminal endoscopic discectomy; lumbar disc herniation; visual analogue scale; early rehabilitation
\end{abstract}

\section{INTRODUCTION}

A lumbar disc herniation is the most common spinal disorder, and it causes widespread medical problems ${ }^{[1,2]}$. The lumbar disc herniation incidence has been rising with the aging population and the changes in the way people work ${ }^{[3]}$. Due to the degeneration of the lumbar intervertebral disc, the fibrous circle is ruptured under external force, which causes nucleus pulposus herniation, leading to peripheral nerve root compression and waist symptoms, such as pain and numbness in the lower extremities ${ }^{[4,5]}$.

A percutaneous transforaminal endoscopic discectomy (PTED) is one effective surgery used for the treatment of a lumbar disc herniation. It was originally used for minimally invasive surgery ${ }^{[6]}$, but nowadays, the PTED

*Corresponding author: Abudourexiti-Tuerhognjiang

Mailing address: Department of Minimally Invasive Spine Therapy, The Sixth Affiliated Hospital of Xinjiang Medical University. Wuxingnan road no.39, Urumchi, Xinjiang, China.

E-mail: ta272@163.com

Received: 25 April 2018 Accepted: 26 June 2018 is widely used in a variety of lumbar degenerative diseases and spinal infectious diseases, achieving a good therapeutic effect ${ }^{[7,8]}$. As a minimally invasive therapy, a PTED has many remarkable advantages, such as a small incision, less surgical complications, short length of stay, safety, small economic burden, and rapid rehabilitation ${ }^{[9,10]}$. Therefore, it has been gradually accepted by more and more surgeons and patients ${ }^{[11]}$. From July 2015 to January 2017, ninety-one patients with lumbar disc herniations were treated with PTEDs that achieved satisfactory curative effects and rapid rehabilitation.

\section{MATERIALS AND METHODS}

\section{General information}

This retrospective study was approved by the Ethics Committee of our hospital, and all of the patients signed informed consent forms. From July 2015 through January 2017, a total of 91 patients with lumbar disc herniations, including 53 males and 38 females (19 to 80 years old, average age $=48.30 \pm 12.70$ years) were selected for this study. L4/5 disc herniations occurred in 
Table 1. Comparison of preoperative VAS score and postoperative (3 days, 3 months and 6 months) VAS score.

\begin{tabular}{|c|c|c|c|c|c|c|}
\hline & \multicolumn{2}{|c|}{ postoperative 3 days } & \multicolumn{2}{|c|}{ postoperative 3 months } & \multicolumn{2}{|c|}{ postoperative 6 months } \\
\hline & low back & leg & low back & leg & low back & leg \\
\hline Preoperative VAS score & $4(3,5)$ & $7(6,7)$ & $4(3,5)$ & $7(6,7)$ & $4(3,5)$ & $7(6,7)$ \\
\hline Postoperative VAS score & $1(0,1)$ & $1(1,2)$ & $1(0,1)$ & $1(1,2)$ & $1(0,1)$ & $1(1,2)$ \\
\hline $\mathrm{Z}$ value & -8.22 & -8.36 & -8.20 & -8.34 & -8.12 & -8.28 \\
\hline$P$ value & $\mathrm{p}<0.05$ & $\mathrm{p}<0.05$ & $\mathrm{p}<0.05$ & $p<0.05$ & $\mathrm{p}<0.05$ & $\mathrm{p}<0.05$ \\
\hline
\end{tabular}

41 cases, L5/S1 disc herniations occurred in 48 cases, and L3/4 disc herniations occurred in 2 cases. The surgeries were completed successfully, and the operation time was 50-145 min, with an average time of 83.40 min. The intraoperative blood loss was $21-53 \mathrm{ml}$, with an average blood loss of $35 \mathrm{ml}$, and the length of stay was 5-13 days, with an average time of 8 days. One patient had cerebrospinal fluid leakage, without severe complications (such as nerve root injuries, abdominal organ injuries, vascular injuries, and intervertebral disc space infections). After the PTED, two cases underwent open surgery due to recurrences, one case underwent open surgery due to bone residue in the spinal canal, and the rate of complications was $4.39 \%$.

\section{Inclusion criteria}

From July 2015 to January 2017, those patients who were admitted to our department and diagnosed with single stage lumbar disc herniations who were willing to undergo PTEDs were included in this study.

\section{Exclusion criteria}

The following patients were excluded from this study: patients with multi-stage lumbar disc herniations, lumbar scoliosis, lumbar disc spondylolisthesis, lumbar spinal stenosis, spinal tuberculosis, spinal tumors, soft tissue infections, a history of lumbar surgery, and hyperparathyroidism. Those patients who took hormone drugs over a long period of time were also excluded.

\section{Surgery}

The patients who met the inclusion criteria underwent lateral plain films of the lumbar vertebrae, computed tomography (CT) plain film scanning of the lumbar intervertebral discs, and magnetic resonance imaging (MRI) scanning of the lumbar vertebrae. The history and physical signs were considered in order to make a definitive diagnosis. All of the patients received nucleus pulposus resections under a transforaminal endoscope, and they were treated with percutaneous endoscopic lumbar discectomies (PELDs) using the transforaminal endoscopic system (joimax $\mathrm{GmbH}$, Karlsruhe, Germany) and a bipolar radiofrequency scalpel (Trigger-Flex; elliquence, Baldwin, NY, USA).

Those patients with L3/4 and L4/L5 lumbar disc herniations were placed in a prone position, the lumbar 4/5 disc space was determined via the positive perspective of the $\mathrm{C}$ arm, and the line of the articular process and joint was determined via the lateral perspective. Then, $12 \mathrm{~cm}$ from the midline of the spinous process, at the lumbar $4 / 5$ level, the puncture point was determined, with $1 \%$ lidocaine used for the local anesthesia, and a Kirschner needle was inserted to the upper margin of the L5. The skin was cut approximately $0.8 \mathrm{~cm}$ at the puncture site, an expansion chamber was inserted, and the working channel was placed into the expansion chamber. The microendoscope operating system and disposable negative pressure drainage tube were connected, and the light was turned on. After inserting the transforaminal endoscope through the working channel, the surrounding fat and ligamentum were removed using grasping forceps, the nerve root was exposed, the protrusion of the nucleus pulposus was removed, and the fibrous ring was ablated via radiofrequency. After a full decompression, the working channel was removed, and the incision was sutured.

General anesthesia was used for those patients with L5/ S1 disc herniations, and it was administered after effective anesthesia. The L5/S1 intervertebral space was determined via the positive perspective of the $\mathrm{C}$ arm, and 2 $\mathrm{cm}$ from the midline of the affected side was determined as the puncture point. The needle was inserted into the medial part of the L5/S1 intervertebral space and the

Table 2. Comparison of postoperative 3 days VAS score and postoperative 3 months and 6 months VAS score.

\begin{tabular}{|c|c|c|c|c|}
\hline & \multicolumn{2}{|c|}{ postoperative 3 months } & \multicolumn{2}{|c|}{ postoperative 6 months } \\
\hline & low back & $\operatorname{leg}$ & low back & $\operatorname{leg}$ \\
\hline Postoperative 3 days VAS score & $1(0,1)$ & $1(1,2)$ & $1(1,2)$ & $1(1,2)$ \\
\hline $\begin{array}{l}\text { Postoperative ( } 3 \text { months, } 6 \\
\text { months) VAS score }\end{array}$ & $1(0,1)$ & $1(1,2)$ & $1(0,1)$ & $1(1,2)$ \\
\hline $\mathrm{Z}$ value & -1.87 & 0 & -1.82 & -1.78 \\
\hline$P$ value & $\mathrm{p}>0.05$ & $\mathrm{p}>0.05$ & $\mathrm{p}>0.05$ & $\mathrm{p}>0.05$ \\
\hline
\end{tabular}


Table 3. Comparison of preoperative ODI index and postoperative (3 days, 3 months and 6 months) ODI index.

\begin{tabular}{llll}
\hline & postoperative 3 days & postoperative 3 months & postoperative 6 months \\
\hline Preoperative ODI index & $67.80 \pm 15.74$ & $67.80 \pm 15.74$ & $67.80 \pm 15.74$ \\
Postoperative ODI index & $30.32 \pm 12.80$ & $17.32 \pm 7.66$ & $16.25 \pm 10.05$ \\
T value & 20.52 & 27.18 & 26.95 \\
P value & $\mathrm{p}<0.05$ & $\mathrm{p}<0.05$ & $\mathrm{p}<0.05$ \\
\hline
\end{tabular}

lower edge of the left lamina. The remaining procedures were the same as those administered after the local anesthesia.

\section{Evaluation of curative effect}

All of the patients were followed up for 6 months. A visual analogue scale (VAS) and the Oswestry Disability Index (ODI) were used to evaluate the pain severity before surgery, and 3 days, 3 months, and 6 months after surgery. The Macnab criteria were used to evaluate the clinical efficacy 6 months after surgery: "excellent" indicated that the symptoms and signs had completely disappeared, and normal activities and work were resumed; "good" indicated that the main symptoms and signs disappeared, the patient could engage in their original work, and no pain medication was required; "medium" indicated that the symptoms and signs were obviously improved, but they still affected work and life activities, and non-steroidal anti-inflammatory drugs (NSIADs) were required; and "bad" indicated that the symptoms and signs had not improved or were even aggravated, and opioids were required. One patient underwent a second operation during the follow-up period.

Statistical analysis

We used the Statistical Package for the Social Sciences version 17.0 for the data analysis. The measured data were analyzed using normal testing and descriptive analyses. If the data showed a normal distribution, they were expressed as the mean \pm standard deviation, and they were analyzed using a paired sample t-test. If the data did not show a normal distribution, they were expressed as the median and interquartile range, and they were analyzed using a non-parametric Wilcoxon rank sum test. P-values of less than 0.05 were considered to be statistically significant.

\section{RESULTS}

Comparisons between the preoperative and postoperative VAS scores

The postoperative (3-day, 3-month, and 6-month) VAS scores for lower back pain were significantly lower than those before surgery $(\mathrm{Z}=-8.22,-8.20$, and -8.12 , respectively, $\mathrm{p}<0.05)$. Additionally, the postoperative (3-day, 3-month, and 6-month) VAS scores for leg pain were significantly lower than those before surgery $(Z=-8.36$,
-8.34 , and -8.28 , respectively, $\mathrm{p}<0.05$ ) (Table 1).

Comparisons among the postoperative VAS scores

The postoperative 3-month VAS score for lower back pain was significantly lower than that of the postoperative 3-day VAS score $(\mathrm{Z}=-1.87, \mathrm{p}<0.05)$. However, there was no significant difference between the postoperative 3-month VAS score for leg pain and the postoperative 3-day VAS score ( $\mathrm{Z}=0, \mathrm{p}>0.05)$. The postoperative 6-month VAS score for lower back pain was lower than that of the postoperative 3-day VAS score, with no statistical significance $(\mathrm{Z}=-1.82, \mathrm{p}>0.05)$. Moreover, there was no significant difference between the postoperative 6-month VAS score for leg pain and the postoperative 3-day VAS score ( $Z=-1.78, p>0.05$ ) (Table 2).

Comparisons between the preoperative and postoperative ODI scores

The postoperative 3-day ODI score was significantly lower than that before surgery $(\mathrm{t}=20.52, \mathrm{p}<0.05)$. In addition, the postoperative 3 -month ODI score was significantly lower than that before surgery $(\mathrm{t}=27.18$, $\mathrm{p}<0.05)$. The postoperative 6-month ODI score was also significantly lower than that before surgery $(t=26.95$, $\mathrm{p}<0.05$ ) (Table 3).

Comparisons among the postoperative ODI scores

The postoperative 3-month ODI score for leg pain was significantly lower than the postoperative 3-day ODI score $(t=10.92, p<0.05)$. The postoperative 6-month ODI score for lower back pain was lower than the postoperative 3-day ODI score, with no statistical significance $(\mathrm{t}=1.96, \mathrm{p}>0.05)$ (Table 4).

Excellent rate of surgery

Accordingtothe Macnabcriteriascore,59 cases(64.83\%) were excellent, 21 cases (23.08\%) were good, 8 cases

Table 4. Comparison of postoperative 3 days ODI index and postoperative 3 months and 6 months ODI index.

\begin{tabular}{lcl}
\hline & $\begin{array}{l}\text { postoperative 3 } \\
\text { days }\end{array}$ & $\begin{array}{l}\text { postoperative 6 } \\
\text { months }\end{array}$ \\
\hline $\begin{array}{l}\text { Postoperative } 3 \\
\text { month ODI index }\end{array}$ & $17.32 \pm 7.66$ & $17.32 \pm 7.66$ \\
$\begin{array}{l}\text { Postoperative 3 day } \\
\text { or 6 month ODI index }\end{array}$ & $30.32 \pm 12.80$ & $16.25 \pm 10.05$ \\
$\begin{array}{l}\text { T value } \\
\text { P value }\end{array}$ & 10.92 & 1.96 \\
\hline
\end{tabular}


(8.79\%) were medium, and 3 cases $(3.30 \%)$ were bad. The total of the excellent and good rates combined was $87.91 \%$

\section{DISCUSSION}

A lumbar disc herniation is the most common spinal disorder, and $68 \%$ of the patients recover after nonsurgical treatment; however, conservative treatment does not work for all patients, so some must have the nucleus pulposus removed via surgery ${ }^{[12]}$. In the past, decompression with a total laminectomy or partial laminectomy using the lumbar posterior approach and open surgery were the standard treatments for the protrusion of a lumbar intervertebral disc. This type of surgery has a high success rate; however, there are also many disadvantages, such as significant trauma, heavy bleeding, damage to the spinal structure, a long postoperative recovery time, and long-term side effects, including lower back and leg pain after surgery ${ }^{[13,14]}$. Due to the improvements in medicine and technology, more and more minimally invasive methods have appeared ${ }^{[15,16]}$. In recent years, the PTED has become widely used in the treatment of lumbar disc herniations, achieving a good effect ${ }^{[7]}$. After the nerve compression is cleared, the calcified intervertebral discs can undergo compression therapy under a transforaminal endoscope ${ }^{[17,18]}$. In the last two years, we applied PTEDs in many patients with good results, which were better than those of open surgery.The comparisons between the preoperativeand postoperative lumbar vertebrae are shown in Figures 1-4. In this study, three cases underwent open surgery due to poor curative effects. One of them had cerebrospinal fluid leakage without severe complications (such as nerve root, abdominal organ, and vascular injuries, and intervertebral disc-space infections). The complication rate was $4.39 \%$, which was lower than the complication rate of traditional open surgery (7\%). The postoperative 3-day VAS score was significantly lower than that before surgery; however, there was no significant difference among the postoperative 3-day, 3-month, and 6-month VAS scores. Therefore, it can be said that the pain relief on the $3^{\text {rd }}$ postoperative day was the same as that during the $3^{\text {rd }}$ and $6^{\text {th }}$ postoperative months. The short-term curative effect was remarkable, and it allowed rapid rehabilitation. Moreover, the postoperative 3-day ODI score was significantly lower than that before surgery, and the postoperative 3-month ODI score was significantly lower than the postoperative 3-day ODI score. However, there was no significant difference between the postoperative 3-month and 6-month scores, which might be related to the scoring items, such as lifting and carrying things, a sedentary lifestyle, sexual activities, and travel. However, the patients were required to rest in bed and avoid severe exercise and sedentariness after the PTED, thus decreasing the ODI scores.

Many factors should be kept in mind during surgery, such as the patient's vital signs and radicular symptoms. Water is the PTED medium, and the procedure can be seen clearly with continuous washing; however, the water pressure should be observed. Too much pressure and excessive time could lead to neck pain and discomfort for the patient. The water temperature should not be too cold, or it will increase the spinal cord response and influence the surgery. In addition, the patient's temperature should be observed, because the incidence of hypothermia is greater than $70 \%$ if the surgery time is more than two hours. This can induce an emergency reaction, reduce the patient's immunity, increase the surgical infection rate, and damage the coagulation mechanism [19,20].

In conclusion, the use of PTEDs for the treatment of lumbar disc herniations was safe and effective, with less trauma, less tissue damage, and a rapid rehabilitation time, which reduced the length of the hospital stay. However, the tissue degeneration of the intervertebral disc is obvious, the loss of intervertebral space is significant, and the recurrence rate is high in patients undergoing excessive nucleus pulposus and fibrous ring removal during the surgery. Therefore, it is suggested that the patient be cautious after surgery, and wear the waist compression bandage for a longer period of time. Weight bearing activities are forbidden for three to six months, and functional exercise should be increased after the fiber ring crevasse is repaired.

\section{CONFLICT OF INTEREST}

None

\section{ACKNOWLEDGEMENTS}

This project was supported by Natural Science Fund Project of Xinjiang uygur autonomous region (No.2017D01C266).

\section{REFERENCES}

1. Thaler, M., Lechner, R., Foedinger, B., Haid, C., Kavakebi, P., Galiano, K., and Obwegeser, A. (2015) Driving reaction time before and after surgery for disc herniation in patients with preoperative paresis. Spine J 15, 918-922

2. Choi, K., Shim, H., Hwang, J., Shin, S., Lee, D., Jung, H., Park, H., and Park, C. (2018) Comparison of surgical invasiveness between microdiscectomy and three different endoscopic discectomy techniques for lumbar disc herniation. World Neurosurg

3. Ma, D., Liang, Y., Wang, D., Liu, Z., Zhang, W., Ma, T., Zhang, L., Lu,X., and Cai,Z. (2013) Trend of the incidence of lumbar disc herniation: decreasing with aging in the elderly. Clin Interv Aging 8, 1047-1050

4. Altinkaya, N., and Cekinmez, M. (2016) Lumbar multifidus 
muscle changes in unilateral lumbar disc herniation using magnetic resonance imaging. Skeletal Radiol. 45, 73-77

5. Strömqvist, F., Strömqvist, B., Jönsson, B., Gerdhem, P., and Karlsson, M. (2015) Outcome of surgical treatment of lumbar disc herniation in young individuals. Bone Joint J 97-B, 1675-1682

6. Henmi, T., Terai, T., Hibino, N., Yoshioka, S., Kondo, K., Goda, Y., Tezuka, F., and Sairyo, K. (2015) Percutaneous endoscopic lumbar discectomy utilizing ventral epiduroscopic observation technique and foraminoplasty for transligamentous extruded nucleus pulposus: technical note. J Neurosurg Spine, 1-6

7. Yang, S., Chen, W., Chen, H., Kao, Y., Yu, S., and Tu, Y. (2014) Extended indications of percutaneous endoscopic lavage and drainage for the treatment of lumbar infectious spondylitis. Eur Spine J 23, 846-853

8. Kim, H., Yudoyono, F., Paudel, B., Kim, K., Jang, J., Choi, J., Chung, S., Kim, J., Jang, I., Oh, S., Park, J., and Lee, S. (2018) Suprapedicular Circumferential Opening Technique of Percutaneous Endoscopic Transforaminal Lumbar Discectomy for High Grade Inferiorly Migrated Lumbar Disc Herniation. Biomed Res Int 2018, 5349680

9. Kondo, M., Oshima, Y., Inoue,H., Takano, Y., Inanami, H., and Koga, H. (2018) Significance and pitfalls of percutaneous endoscopic lumbar discectomy for large central lumbar disc herniation. J Spine Surg 4, 79-85

10. Zhou, Y., Chen, G., Bi, D., and Chen, X. (2018) Shortterm clinical efficacy of percutaneous transforaminal endoscopic discectomy in treating young patients with lumbar disc herniation. J Orthop Surg Res 13, 61

11. Tacconi, L., Baldo, S., Merci, G., and Serra, G. (2018) Transforaminal percutaneous endoscopic lumbar discectomy: outcome and complications in 270 cases. J Neurosurg Sci

12. Davis,N.,Hourigan,P.,andClarke,A.(2017)Transforaminal epidural steroid injection in lumbar spinal stenosis: an observational study with two-year follow-up. Br J Neurosurg 31, 205-208

13. Schwender, J., Holly, L., Rouben, D., and Foley, K. (2005) Minimally invasive transforaminal lumbar interbody fusion (TLIF): technical feasibility and initial results. J Spinal Disord Tech 18 Suppl, S1-6

14. Carilli, S., Oktenoglu, T., and Ozer, A. (2006) Openwindow laparotomy during a transperitoneal approach to the lower lumbar vertebrae: new method for reducing complications. Minim Invasive Neurosurg 49, 227-229

15. Kamper, S., Ostelo, R., Rubinstein, S., Nellensteijn, J., Peul, W., Arts, M., and van Tulder, M. (2014) Minimally invasive surgery for lumbar disc herniation: a systematic review and meta-analysis. Eur Spine J 23, 1021-1043

16. Kogias, E., Franco Jimenez, P., Klingler, J., and Hubbe, U. (2015) Minimally invasive redo discectomy for recurrent lumbar disc herniations. J Clin Neurosci 22, 1382-1386

17. Gioffre', G., Impusino, A., and Tacconi, L. (2017) Retroperitoneal hematoma after minimally invasive lumbar discectomy: is the percutaneous endoscopic approach really safe? J Neurosurg Sci

18. Shiboi, R., Oshima, Y., Kaneko, T., Takano, Y., Inanami, H., and Koga, H. (2017) Different operative findings of cases predicted to be symptomatic discal pseudocysts after percutaneous endoscopic lumbar discectomy. J Spine Surg 3, 233-237

19. Bernard, H. (2013) Patient warming in surgery and the enhanced recovery. Br J Nurs 22, 319-320, 322-315

20. Munday, J., Hines, S., and Chang, A. (2013) Evidence utilisation project: Management of inadvertent perioperative hypothermia. The challenges of implementing best practice recommendations in the perioperative environment. Int J Evid Based Healthc 11, 305-311 